\title{
Estudo de viabilidade econômica de construção de um hotel na cidade de Vassouras-RJ
}

\author{
Economic feasibility study for the construction of a hotel in the city of Vassouras-RJ
}

Lucelia Fonseca Rocha, Felipe Ribeiro Nogueira, Marco Antônio Vaz Capute, Luiz Felipe Caramez Berteges.

Como citar esse artigo. Rocha, LF; Nogueira, FR; Capute, MAV; Berteges, LFC. Estudo de viabilidade econômica de construção de um hotel na cidade de Vassouras-RJ. Revista Teccen. 2021 Jul./Dez.; 14 (2): 02-06.

\begin{abstract}
Resumo
No presente trabalho, estudou-se a viabilidade econômica da construção de um hotel no Município de Vassouras, região do Vale do Café. Como consequência da pandemia do COVID-19 no Brasil o turismo foi um dos setores mais afetados no ano de 2020 trazendo impactos socioeconômicos no país. O objetivo principal deste trabalho foi analisar a viabilidade econômica de construção de um hotel. Para o desenvolvimento da análise econômica foi realizado o levantamento de informações na rede hoteleira do município de Vassouras e orçamentos perante empresas do seguimento. Estas informações foram subsídios para a concretização do planejamento econômico. Realizou-se projeções de fluxo de caixa com uma taxa média de ocupação, concluindo a análise de gastos e faturamento anual. Após análise dos resultados da Taxa Interna de Retorno (TIR), Taxa Mínima de Atratividade (TMA), PAYBACK e Valor Presente Líquido (VPL) obtidos, permitiu-se concluir que o projeto tem viabilidade econômica favorável para ser realizado.

Palavras-Chave: Hotelaria; Análise; Turismo; Vale do Café.
\end{abstract}

\begin{abstract}
In the present work, the economic feasibility of building a hotel in the municipality of Vassouras, region of Vale do Café was studied. As a result of the COVID-19 pandemic in Brazil, tourism was one of the most affected sectors in 2020 , bringing impacts socioeconomic conditions in the country. The main objective of this work was to analyze the economic feasibility of building a hotel. For the development of the economic analysis, a survey of information on the hotel network in the city of Vassouras and budgets from companies in the segment was carried out. This information was a subsidy for the implementation of economic planning. Cash flow projections were carried out with an average occupancy rate, completing the annual expenditure and billing analysis. After analyzing the results of the Internal Rate of Return (IRR), Minimum Attractiveness Rate (TMA), PAYBACK and Net Present Value (NPV) obtained, it was concluded that the project has favorable economic viability to be carried out.
\end{abstract}

Keywords: Hospitality; Analysis; Tourism; Vale do Café.

\section{Introdução}

Em meados de 2020, o mundo entrou em colapso devido a pandemia da COVID-19. Os impactos gerados no Brasil atingiram, sem precedentes, a sociedade, a economia, a política e a cultura do país (FIOCRUZ, 2021). De acordo com a nota produzida pela Secretaria de Política Econômica do Ministério da Economia sobre as medidas de combate, em 2020, uma das áreas mais afetadas foi a economia brasileira, por consequência das medidas preventivas adotadas pelo Ministério da Saúde, como lockdown, distanciamento social, protocolos de higienização entre outros. A eclosão da pandemia, gerou quedas no real e aumento no dólar, causando inflação, aumentando a produção e a logística no país.

A rede hoteleira fora desafiada, havendo demissões, reduções salariais e de jornada de trabalho. Estabelecimentos indo à falência e em vários momentos todos os tipos de serviços foram obrigados a fechar momentaneamente. Visto isso, os hotéis inovaram, priorizando as normas preventivas no combate à Covid-

Afiliação dos autores: 
19, investindo na higienização severamente. Com isso, este ramo tem se desenvolvido, destacando-se no mercado de trabalho devido ao seu crescimento no marketing digital, atraindo os clientes através conforto, localização e lazer.

O objetivo deste presente trabalho foi avaliar a viabilidade econômica de construção de um hotel na cidade de Vassouras, visando analisar o setor de turismo do município e da região do Vale do Café obedecendo aos protocolos para conter a pandemia. Justifica-se o desenvolvimento deste trabalho, visto que o turismo na região do Vale do Café está ganhando seu espaço na mídia digital e os turistas têm procurado o contato com a natureza e a calmaria do interior, na qual a região se enquadra nestes quesitos. Segundo o IBGE, a população estimada de Vassouras em 2021 é de 37.262 habitantes. Em visita a Secretaria de Turismo da região, foi informado que a média de turistas chega a 900 pessoas por mês, o que intensifica na tomada de decisão para realização do projeto.

\section{Materiais e Métodos}

Através de uma revisão bibliográfica, analisouse um plano de negócios e um estudo de caso no ramo hoteleiro, buscando informações no que diz respeito à viabilidade econômica para construção de hotéis.

Inicialmente foi necessário realizar um estudo da localização para implantação do hotel e os principais pontos que compõem o município. Realizou-se uma pesquisa de dados na Secretaria de Turismo da cidade de Vassouras em busca do quantitativo de hotéis no município, o fluxo de turistas e suas finalidades ao visitar a região obtendo-se as informações necessárias que impactam no estudo, tais como cadastros, impostos etc.

Para analisar a viabilidade econômica, foram orçados os valores de investimento inicial, equipamentos, mobílias, impostos e gastos operacionais. Realizada a coleta de dados, foi elaborada uma planilha em Excel para a inserção das informações obtidas para uma verificação minuciosa de gastos, custos, despesas, dentre outros dados.

Com o estudo do público-alvo e taxa de ocupação mensal, criou-se um cenário estimando a receita mensal média da empresa, incluindo outras maneiras de obter receitas, como: vendas com suvenir, amenidades, aluguéis de lojas e terceirização de restaurantes. Com o preenchimento dos dados para o fluxo de caixa, foi possível o estudo dos investimentos utilizando indicadores, tais como a Taxa Interna de Rentabilidade (TIR), Taxa Mínima de Atratividade (TMA), PAYBACK e (Valor Presente Líquido) VPL.

De acordo com Mantelli (2017), a TIR é o índice que mede a rentabilidade do investimento. Quando a porcentagem da TIR estiver com o valor acima do valor da TMA proposto, o projeto se torna viável. Pode ser apresentado pela seguinte equação:

$$
\sum_{j=0}^{n} F C_{J} \cdot\{1-(1+i)\}^{j}=0
$$

Para Schroeder et al. (2005), a taxa mínima de atratividade é o retorno mínimo exigido pelo investidor financeiramente. Determina-se através da porcentagem estipulada pelo investidor para obter o capital inicial de volta.

Segundo Mantelli (2017), o Payback, traduzido da língua inglesa como retorno, é o tempo de recuperação do capital investido e tem por definição algébrica a seguinte equação:

$$
\text { Payback }=F C_{0} / F C_{\text {médio }}
$$

O VPL caracteriza-se como o indicador que demonstra com mais realidade o retorno futuro do investimento. Este método reconhece o valor no tempo, pois utiliza o valor presente das entradas e o valor presente das saídas (Mantelli, 2017). Pode ser representada pela forma algébrica abaixo:

$$
V P L(i)=\sum_{i=0}^{n} F C_{j} /(1+i)^{j}
$$

Ainda como referência de estudo, utilizou-se dados da Associação Brasileira da Industria de Hotéis (ABIH), que tem como principais finalidades o estudo, a consultoria e a resolução de problemas no setor hoteleiro. Cabe destacar que a cidade de Vassouras foi contemplada por um evento da $\mathrm{ABIH}$ realizado no Centro de Convenções General Sombra de Vassouras para conhecimento na área de marketing e negócios, intensificando a venda do turismo na região do Vale do Café, proporcionando acessibilidade, credibilidade e visibilidade para um mercado e região que está tendo um crescimento abundante (ABIH, 2021).

\section{Resultados e Discussão}

\section{Localização}

Conforme já mencionado, o projeto prevê a construção do hotel na cidade turística e histórica de Vassouras, composta por diversos parâmetros turísticos, públicos e da área da saúde. Denominado de Hotel Vale Imperial, o plano estrutural conta com 100 acomodações divididas entre quartos duplos e triplos, sauna, piscina, 2 restaurantes gourmet temáticos, salâo de beleza, spa, salão para eventos e salas para reuniões. A iniciativa de construção conta com um empresário local, que idealiza 
a cidade histórica com os fundamentos de manter este padrão caracterizado por ser um local acolhedor, com transmissão de paz e cultura.

\section{Público-alvo}

O estudo do público-alvo teve como embasamento os locais públicos e históricos denominados anteriormente na localização sabendo que existe grande demanda para estes pontos na cidade. Com isso, definiuse que são: companhias ou grupos que estejam em um evento/reunião no Centro de Convenções; familiares dos universitários; acompanhantes de pacientes que estejam nos Hospitais aos arredores e pessoas que buscam por descanso e conforto.

Segundo informado pela Secretaria de Turismo de Vassouras, aproximadamente 900 pessoas visitam a cidade mensalmente, dentre elas, turistas que correspondem a $60 \%$ dos visitantes, $20 \%$ são caracterizados por pessoas que estão na cidade para fins de trabalho/negócios e os demais $20 \%$ destinam-se a pessoas em busca de lazer e eventos.

\section{Estudo financeiro}

O estudo se inicia através de toda análise de custos variáveis e fixos. O CAPEX EXPENDITURE, traduzido da língua inglesa como despesas de capitais, apresenta os investimentos em bens. Para desenvolvimento do cenário econômico, inicialmente avaliou-se a análise da compra de um lote no valor de $\mathrm{R} \$ 10.000 .000,00$ com 11 mil $\mathrm{m}^{2}$, obra de $6 \mathrm{mil} \mathrm{m}^{2}$ no total de $\mathrm{R} \$ 18,000.000,00$ de construção. Os equipamentos e mobílias necessários ao projeto totalizaram o valor de $\mathrm{R} \$ 407.329,00$ e $\mathrm{R} \$$ $\mathrm{R} \$ 730.890,00$. Os dados foram obtidos através de pesquisa de valores de equipamentos e mobília com quantidades e valores estimados, totalizando um investimento inicial de R \$ 29.138.219,00.

Para execução de rotina em um hotel, são necessários gastos fixos com colaboradores, energia elétrica, água, internet, material de escritório, ou seja, tudo aquilo que é necessário para manter um estabelecimento funcionando. Nas Tabelas 1 e 2 são apresentados os referidos gastos. O OPEX- EXPENDITURE, é o demonstrativo de gastos operacionais da empresa.

Estes gastos são variáveis, devido as oscilações mensais, portanto o cálculo estimado é de R $\$ 175.509,33$ ao mês e R\$2.106.111,96 ao ano.

\section{Receita}

O cálculo da receita foi elaborado de forma simples, através da quantidade de pessoas que se instalam nos hotéis do município, foi desenvolvido uma média através da taxa de ocupação. A taxa e o valor
Tabela 1. Gastos com colaboradores.

\begin{tabular}{|c|c|c|c|c|c|c|c|c|}
\hline \multirow{2}{*}{$\begin{array}{c}\text { Colaborador } \\
\text { Administrativo }\end{array}$} & \multirow{2}{*}{$\begin{array}{c}\text { Quant. } \\
1\end{array}$} & \multicolumn{2}{|c|}{ Valor } & \multirow{2}{*}{$\begin{array}{l}\text { Imposto } \\
68,2 \%\end{array}$} & \multicolumn{2}{|c|}{ Valor Imposto } & \multicolumn{2}{|r|}{ Total } \\
\hline & & RS & $1.715,00$ & & RS & $1.169,63$ & $\mathrm{R} \$$ & $2.884,63$ \\
\hline $\begin{array}{l}\text { Almoxarifado/estoqu } \\
\text { e/compras }\end{array}$ & 1 & RS & $1.400,00$ & $68,2 \%$ & RS & 954,80 & $\mathrm{R} \$$ & $2.354,80$ \\
\hline Assistente de reservas & 1 & RS & $1.500,00$ & $68,2 \%$ & RS & $1.023,00$ & $\mathrm{R} \$$ & $2.523,00$ \\
\hline Camareira & 4 & RS & $1.400,00$ & $68,2 \%$ & RS & 954,80 & R\$ & $9.419,20$ \\
\hline $\begin{array}{l}\text { Coord.Comercial e } \\
\text { Vendas }\end{array}$ & 1 & RS & $4.000,00$ & $68,2 \%$ & RS & $2.728,00$ & R\$ & $6.728,00$ \\
\hline $\begin{array}{l}\text { Coord. de Gerência } \\
\text { de Gente e Finanças }\end{array}$ & 1 & RS & $4.000,00$ & $68,2 \%$ & RS & $2.728,00$ & $\mathrm{R} \$$ & $6.728,00$ \\
\hline $\begin{array}{l}\text { Coord. de Logística e } \\
\text { Operacional }\end{array}$ & 1 & RS & $4.000,00$ & $68,2 \%$ & RS & $2.728,00$ & $\mathrm{R} \$$ & $6.728,00$ \\
\hline Faturista & 1 & RS & $1.800,00$ & $68,2 \%$ & RS & $1.227,60$ & $\mathrm{R} \$$ & $3.027,60$ \\
\hline Lavanderia & 2 & RS & $1.200,00$ & $68,2 \%$ & RS & 818,40 & $\mathrm{R} \$$ & $4.036,80$ \\
\hline Líder/Gerente & 1 & RS & $7.000,00$ & $68,2 \%$ & RS & $4.774,00$ & $\mathrm{R} \$$ & $11.774,00$ \\
\hline Manobrista & 2 & RS & $1.260,00$ & $68,2 \%$ & RS & 859,32 & R\$ & $4.238,64$ \\
\hline Manutenção & 2 & RS & $1.800,00$ & $68,2 \%$ & RS & $1.227,60$ & $\mathrm{R} \$$ & $6.055,20$ \\
\hline Recepcionista & 5 & RS & $1.700,00$ & $68,2 \%$ & RS & $1.159,40$ & $\mathrm{R} \$$ & $14.297,00$ \\
\hline Serviços gerais & 2 & RS & $1.265,00$ & $68,2 \%$ & RS & 862,73 & $\mathrm{R} \$$ & $4.255,46$ \\
\hline Total mês & 25 & R\$ & $4.040,00$ & $68,2 \%$ & & $3.215,28$ & RS & 85.050,33 \\
\hline Total ano & & R\$4 & 88.480,00 & & R\$2 & $88.583,36$ & R\$1. & $020.603,96$ \\
\hline
\end{tabular}

Fonte: Autores, 2021.

\begin{tabular}{c|c}
$\begin{array}{c}\text { Tabela 2. Custos gerais. } \\
\text { Custos }\end{array}$ & Valor \\
\hline Água & $\mathrm{R} \$ 13.800,00$ \\
Assinatura de canais & $\mathrm{R} \$ 1.000,00$ \\
Café da manhã & $\mathrm{R} \$ 21.600,00$ \\
Contabilidade & $\mathrm{R} \$ 500,00$ \\
Energia elétrica & $\mathrm{R} \$ 27.069,00$ \\
Internet/telefone & $\mathrm{R} \$ 500,00$ \\
Material de escritório & $\mathrm{R} \$ 300,00$ \\
Material de limpeza & $\mathrm{R} \$ 25.000,00$ \\
Sistema & $\mathrm{R} \$ 690,00$ \\
\hline Total mês & $\mathbf{R} \$ \mathbf{9 0 . 4 5 9 , 0 0}$ \\
\hline Total ano & $\mathbf{R} \$ \mathbf{1 . 0 8 5 . 5 0 8 , 0 0}$ \\
& Fonte: Autores, 2021.
\end{tabular}

de estadia, foram definidas sendo $40 \%$ de ocupação e R $\$ 800,00$ a acomodação, independente se terá alta ou baixa temporada, a fim de calcular a média mensal de estadias no hotel. O hotel será composto por 100 quartos e estará em funcionamento todo o mês, portanto calcula-se: 
Receita $=R \$ 800,00$ (estadia) $\times 100$ (quant.de quartos disponíveis) $\mathrm{x} 0,40$

(taxa de ocupação estimada) x 30 (quant.de dias em funcionamento $)=\mathrm{R} \$ 960.000,00 /$ mês

Obtendo uma receita anual de $\mathrm{R} \$ 11.520 .000,00$. O projeto conta com aluguéis de estabelecimentos no valor mensal de $\mathrm{R} \$ 37.500,00$ e $\mathrm{R} \$ 450.000,00$ anual conforme tabela 3. Foi considerado que os anos 1,2 e 3 serão feitas a construção e a montagem dos equipamentos.

Tabela 3. Receitas.

\begin{tabular}{|c|c|c|c|c|}
\hline Ano & Quartos & $\begin{array}{c}\text { Aluguel } \\
\text { restaurante }\end{array}$ & $\begin{array}{l}\text { Aluguéis } \\
\text { de lojas }\end{array}$ & $\begin{array}{c}\text { Aluguel salão } \\
\text { de festas }\end{array}$ \\
\hline ANO 1 & 0 & 0 & 0 & 0 \\
\hline ANO 2 & 0 & 0 & 0 & 0 \\
\hline ANO 3 & 0 & 0 & 0 & 0 \\
\hline ANO 4 & $\mathrm{R} \$ 11.520 .000,00$ & $\mathrm{R} \$ 168.000,00$ & $\begin{array}{c}\mathrm{R} \$ \\
90.000,00\end{array}$ & $\begin{array}{c}\mathrm{RS} \\
192.000,00\end{array}$ \\
\hline ANO 5 & $\mathrm{R} \$ 11.520 .000,00$ & $\begin{array}{c}\mathrm{R} \$ \\
168.000,00\end{array}$ & $\begin{array}{c}\mathrm{RS} \\
90.000,00\end{array}$ & $\begin{array}{c}\mathrm{RS} \\
192.000,00\end{array}$ \\
\hline ANO 6 & $\mathrm{R} \$ 11.520 .000,00$ & $\begin{array}{c}\mathrm{R} \$ \\
168.000,00\end{array}$ & $\begin{array}{c}\mathrm{R} \$ \\
90.000,00\end{array}$ & $\begin{array}{c}\mathrm{R} \$ \\
192.000,00\end{array}$ \\
\hline ANO 7 & $\mathrm{R} \$ 11.520 .000,00$ & $\begin{array}{c}\mathrm{R} \$ \\
168.000,00\end{array}$ & $\begin{array}{c}\mathrm{R} S \\
90.000,00\end{array}$ & $\begin{array}{c}\mathrm{R} \$ \\
192.000,00\end{array}$ \\
\hline ANO 8 & $\mathrm{R} \$ 11.520 .000,00$ & $\begin{array}{c}\mathrm{R} \$ \\
168.000,00\end{array}$ & $\begin{array}{c}\mathrm{RS} \\
90.000,00\end{array}$ & $\begin{array}{c}\mathrm{R} \$ \\
192.000,00\end{array}$ \\
\hline ANO 9 & $\mathrm{R} \$ 11.520 .000,00$ & $\begin{array}{c}\mathrm{R} \$ \\
168.000,00\end{array}$ & $\begin{array}{c}\mathrm{RS} \\
90.000,00\end{array}$ & $\begin{array}{c}\mathrm{R} \$ \\
192.000,00\end{array}$ \\
\hline ANO 10 & $\mathrm{R} \$ 11.520 .000,00$ & $\begin{array}{c}\mathrm{R} \$ \\
168.000,00\end{array}$ & $\begin{array}{c}\mathrm{RS} \\
90.000,00\end{array}$ & $\begin{array}{c}\mathrm{RS} \\
192.000,00\end{array}$ \\
\hline ANO 11 & $\mathrm{R} \$ 11.520 .000,00$ & $\begin{array}{c}\mathrm{R} \$ \\
168.000,00\end{array}$ & $\begin{array}{c}\mathrm{R} \$ \\
90.000,00\end{array}$ & $\begin{array}{c}\mathrm{R} \$ \\
192.000,00\end{array}$ \\
\hline ANO 12 & $\mathrm{R} \$ 11.520 .000,00$ & $\begin{array}{c}\mathrm{R} \$ \\
168.000,00\end{array}$ & $\begin{array}{c}\mathrm{R} S \\
90.000,00\end{array}$ & $\begin{array}{c}\mathrm{RS} \\
192.000,00\end{array}$ \\
\hline ANO 13 & $\mathrm{R} \$ 11.520 .000,00$ & $\begin{array}{c}\mathrm{R} \$ \\
168.000,00\end{array}$ & $\begin{array}{c}\mathrm{R} S \\
90.000,00\end{array}$ & $\begin{array}{c}\mathrm{R} \$ \\
192.000,00\end{array}$ \\
\hline ANO 14 & $\mathrm{R} \$ 11.520 .000,00$ & $\begin{array}{c}\mathrm{R} \$ \\
168.000,00\end{array}$ & $\begin{array}{c}\mathrm{RS} \\
90.000,00\end{array}$ & $\begin{array}{c}\mathrm{R} \$ \\
192.000,00\end{array}$ \\
\hline ANO 15 & $\mathrm{R} \$ 11.520 .000,00$ & $\begin{array}{c}\mathrm{R} \$ \\
168.000,00\end{array}$ & $\begin{array}{c}\mathrm{R} \$ \\
90.000,00\end{array}$ & $\begin{array}{c}\mathrm{R} \$ \\
192.000,00\end{array}$ \\
\hline
\end{tabular}

Fonte: Autores, 2021.

\section{Fluxo de caixa e PAYBACK}

O fluxo de caixa representa todas as entradas e saídas de uma determinada empresa. É o planejamento financeiro de todo o projeto, apresentado na tabela 4 . Caracteriza-se por determinar receitas e gastos futuros ao longo do tempo. Para realizar o fluxo de caixa basta somar as receitas e diminuir as despesas.

O PAYBACK é uma importante ferramenta para mensuração, para calcular em quanto tempo haverá o retorno financeiro, como mostra a tabela 4, o PAYBACK será a partir do ano 7 do projeto.

Tabela 4. Fluxo de caixa.

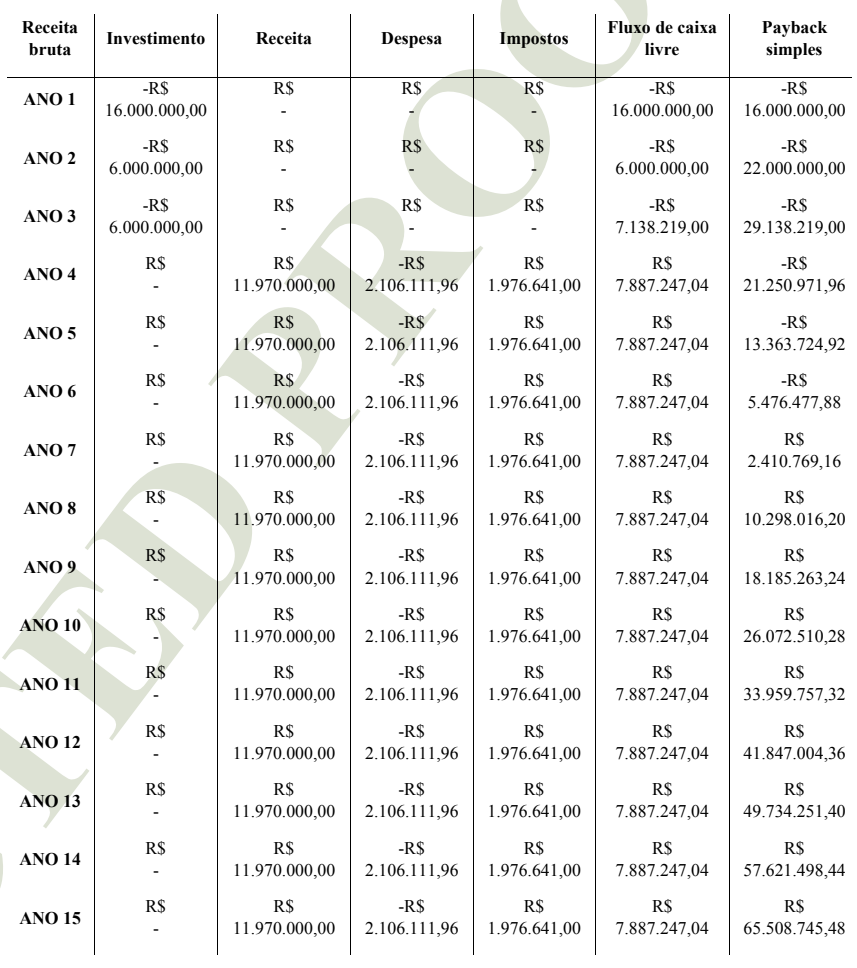

Fonte: Autores, 2021.

\section{Lucro real e presumido}

O lucro real é uma forma de tributação que é sobreposto no valor do resultado líquido, ou seja, receitas menos despesas. Nesta tributação o COFINS é de $7,6 \%$ e o PIS é de $1,65 \%$.

O lucro presumido é a tributação que utiliza o imposto no valor do faturamento total. Neste tipo de imposto o COFINS é de 3\% e PIS 0,65\%. Para escolher o tipo de tributação adequado para seu tipo de empresa, é necessário aplicar as duas formas de tributação sobreo fluxo de caixa feito e efetuar a comparação dos valores. Neste projeto, foi utilizado o lucro presumido é ISS $2 \%$.

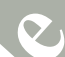




\section{Taxa interna de Retorno - TIR}

O cálculo utilizado foi o fluxo de caixa livre e o PAYBACK. Encontrando-se a TIR como 20\%. Sendo o TMA deste projeto definido pelo seu investidor de $10 \%$, a TIR de 20\% mostra-se vantajosa para o investidor.

\section{Valor Presente Líquido - VPL}

O valor de VPL é outra ferramenta para análise econômica, onde caso o valor encontrado seja positivo demonstra que o projeto não trará prejuízos, e para o projeto o valor de VPL encontrado foi de R $\$$ 21.494.593,41.

\section{Considerações finais}

O principal objetivo deste estudo, foi avaliar a implantação de um hotel no Município de VassourasRJ, região do Vale do Café. As receitas analisadas neste fluxo foram contabilizadas no valor de R $\$ 11.970 .000,00$ com a taxa de ocupação de $40 \%$, aluguéis de lojas e restaurantes. As despesas foram calculadas baseadas na taxa de ocupação que corresponde as receitas, totalizando R \$ 2.106.111,96. Obtendo um lucro bruto de $\mathrm{R} \$ 9.863 .888,04$.

Aplicando a tributação por presunção no lucro bruto e aplicando os impostos IRPJ, CSSL, Adicional de imposto de renda, concluiu de fato que o total de impostos em porcentagem a serem pagos são de $16,51 \%$, ou seja, R\$ 1.976.641,00. Efetuando o cálculo do lucro bruto menos os impostos obtêm-se um total de $\mathrm{R} \$$ 7.887.247,04 de caixa livre.

Desta forma, após análise dos resultados da Taxa Interna de Retorno (TIR) em comparação com a Taxa Mínima de Atratividade (TMA), os resultados do PAYBACK e Valor Presente Líquido (VPL) obtidos, permitiu-se concluir que o projeto é viável para ser implementado

\section{Referências}

ABIH, de 9 de junho de 2021. Programa de capacitação da ABIH-RJ passará pelo Vale do Café. Recuperado em 28 de setembro de 2021 de https://abihrj. com.br/noticias/programa-de-capacitacao-da-abih-rj-passara-pelo-vale-docafe/.

FIOCRUZ. Impactos sociais, econômicos, culturais e políticos da pandemia. Recuperado em 11 de agosto de 2021 de https://portal.fiocruz.br/impactossociais-economicos-culturais-e-politicos-da-pandemia.

GOVERNO FEDERAL, de 17 de abril de 2020. Nota informativa-Medidas de Combate aos Efeitos Econômicos da COVID-19. Recuperado em 13 de setembro de 2021 de https://www.gov.br/economia/pt-br/centrais-deconteudo/publicacoes/notas-informativas/2020/nota-informativa-medidasfiscais-coronavirus-final-17 04.pdf

IBGE. Cidade e Estados - Vassouras. Recuperado em 24 de agosto de 2021

de https://www.ibge.gov.br/cidades-e-estados/rj/vassouras.html.

Mantelli, J. V., (2017). Plano de Negócios - Hotel do Vale. Recuperado em 11 de agosto de 2021 de https://www.univates.br/bdu/bitstream/10737/1650/ 1/2017ValmorMantelliJunior.pdf

Schroeder, T. J., Shroeder I., Da Costa, P. R. \& Shinoda, C. (2005). O Custo de Capital como Taxa Mínima de Atratividade na Avaliação de Projetos de Investimento. Revista de Gestão Industrial, 01. Recuperado em 23 de setembro de 2021 de https://periodicos.utfpr.edu.br/revistagi/article/ view/163/. 